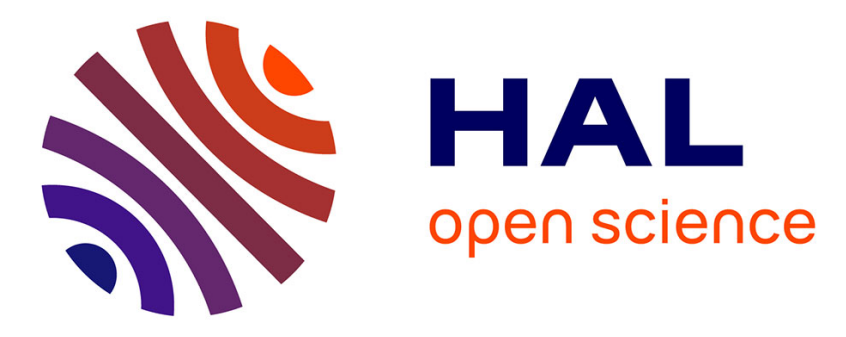

\title{
Robust Interval Luenberger Observer-Based State Feedback Control: Application to a Multi-DOF Micropositioner
}

Mounir Hammouche, Abdenbi Mohand Ousaid, Philippe Lutz, Micky

Rakotondrabe

\section{To cite this version:}

Mounir Hammouche, Abdenbi Mohand Ousaid, Philippe Lutz, Micky Rakotondrabe. Robust Interval Luenberger Observer-Based State Feedback Control: Application to a Multi-DOF Micropositioner. IEEE Transactions on Control Systems Technology, 2018, 27 (6), pp.2679 - 2679. 10.1109/TCST.2018.2865767 . hal-02399259

\section{HAL Id: hal-02399259 \\ https://hal.science/hal-02399259}

Submitted on 9 Dec 2019

HAL is a multi-disciplinary open access archive for the deposit and dissemination of scientific research documents, whether they are published or not. The documents may come from teaching and research institutions in France or abroad, or from public or private research centers.
L'archive ouverte pluridisciplinaire HAL, est destinée au dépôt et à la diffusion de documents scientifiques de niveau recherche, publiés ou non, émanant des établissements d'enseignement et de recherche français ou étrangers, des laboratoires publics ou privés. 


\title{
Robust interval Luenberger observer-based state feedback control: application to a multi-DOF micropositioner
}

\author{
Mounir Hammouche, Abdenbi Mohand-Ousaid, Philippe Lutz, Member, IEEE, and Micky \\ Rakotondrabe, Member, IEEE
}

\begin{abstract}
Controlling multi-DOF (Degree of Freedom) micropositioning systems always represents great challenge because of the high sensitivity to the environment at this scale and the cross-coupling effects present between the different axes. A robust Luenberger observer-based state feedback design using interval analysis and regional pole assignment technique are introduced to control such systems. This robust control design keeps the same structure of the classical state-feedback with the usual Luenberger observer. However, the synthesises of the observer and the feedback controller are performed by means of interval techniques to find the set of gains that are robust against system uncertainties and that satisfy some predefined performances. For this matter, an algorithm based on Set Inversion Via Interval Analysis (SIVIA) combined with interval eigenvalues computation is proposed to find these robust gains. The control approach is validated in simulation and then tested experimentally to control a multi-DOF positioning structure.
\end{abstract}

Index Terms-Luenberger observer-based state feedback, interval models, SIVIA, micropositioner.

\section{INTRODUCTION}

During the last decades, the design of micropositioning systems based on smart materials such as piezoelectric actuators have gained much attention [1]. Unfortunately the micropositioning systems are subjected to several uncertainties produced by various factors such as vibrations, ambient temperature, sensors limitation, dynamics modeling, and the nonlinear characteristics of the used piezoelectric actuators (hysteresis, time varying parameters, creep, etc), which make the control of these structures not a trivial task. These factors must be considered during the control design by including enough robustness to the controller otherwise the control system will fail.

There are various methods to control piezoelectric-based actuators including real-time adaptive, nonlinear techniques and robust approaches that take into account these uncertainties [1], [2]. There are also other robust approaches on the basis of interval linear models that embrace the nonlinearities and the uncertainties of the system [3], [4]. These interval techniques present a very interesting method to represent system uncertainties and to synthesize a robust controller [1]. However, the

The authors are with the Automatic Control and Micro-Mechatronic Systems Department, AS2M. FEMTO-ST Institute, UMR CNRS 6174 - UBFC / ENSMM / UTBM

24 rue Alain Savary, 25000 Besançon, France.

e-mail: \{mounir.hammouche, abdenbi.mohand, plutz, mrakoton \}@femto-st.fr. Paper type: Brief paper. First submission: Septembre 2017. previous studies of interval control design focus on modeling the processes by an interval transfer function representation which is not well adapted to multivariable systems. Indeed, the recent advance on the design of actuators with multiDOF especially for micro/nano-world raises the problem of designing robust controller for multivariable systems [1], [2]. Therefore, the state-space based interval modeling which is adequate to multivariable systems is studied in this paper.

The robust state-feedback controller synthesis for interval state-space models using pole placement technique has been considered in several works [5], [6], [7]. However the above works focus only on the study of robust state-feedback control design using interval Ackermann's equation and closed-loop characteristic polynomial. Furthermore, they are limited to systems with state and input matrices of special structures as explained in [5].

This paper addresses the problem of robust Luenberger observer-based state feedback design to control multi-DOF micropositioner under system uncertainties described by an interval state-space model. Indeed, we propose to employ the interval analysis techniques to find easily the set of robust gains for both the observer and the controller, separately, that ensure the stability and satisfy the desired performances. For this purpose, a recursive algorithm has been introduced to obtain the set of robust gains using regional pole assignment techniques and interval eigenvalue computation. The proposed design approach is guaranteed for a large class of interval linear systems. Finally, the proposed control strategy is validated experimentally using a multi-DOF positionner stage based on a monolithic skeleton driven by piezoelectric actuators.

The paper is organized as follows. Section 2 is dedicated to brief preliminaries on interval eigenvalues computation. Section 3 presents the structure of the observer-based state feedback scheme and problem formulation. The experimental setup and system identification are given in section 4 . Whereas, a simulation validation and experimental results are discussed in Section 5 and 6. Finally, conclusion is given in Section 7.

\section{INTERVAL EIGENVALUE COMPUTATION}

This section brings a brief preliminaries on interval eigenvalues computation. The preliminaries of interval analysis and interval matrix theory can be found in [8].

The interval eigenvalue $\Lambda$ of an interval matrix is defined as the set of all eigenvalues over all $A \in \mathbf{A}$, that is [6], 


$$
\Lambda(\boldsymbol{A})=\{\lambda+i \mu \mid \exists A \in \boldsymbol{A}, \exists x \neq 0: A x=(\lambda+i \mu) x)\}
$$

The interval eigenvalue computation can be summarized by estimating an outer bound in which all eigenvalues of the matrix $A \in \mathbf{A}$ are bounded.

Recent advances in interval analysis computation have provided a new opportunity to estimate easily an outer bound of the interval eigenvalue for any class of interval matrices including real symmetric and asymmetric interval matrices. In the case of a real symmetric interval matrix, it is worthy to note that the matrix $\mathbf{A}^{\mathrm{s}}$ has only real eigenvalues.

To estimate the outer bound of the interval eigenvalue, there are several methods existing in the literature. In [9] and [10], the authors proposed some approaches to provide the exact bound for the interval eigenvalue, however, these methods are based on hard assumptions which are not easy to verify as explained in [11]. Furthermore, in [12] and [13], the authors employed the Taylor expansion and Perturbation theorems to estimate the outer bound for real and complex interval matrices. Recently, cheap formulas to estimate the outer bound of the interval eigenvalue are introduced by Rohn in [14] and by Hladîk in [11] for a class of symmetric and asymmetric interval matrices, respectively.

Moreover, there is another interesting method to estimate the outer bound of the interval eigenvalue called the vertex approach introduced recently in [15] based on the work of [16]. The basic concepts of the vertex approach are the computation of the eigenvalues of all exposed edges of the interval matrix and the use of the convex hull function to find a convex polygon that enclose all possible eigenvalues. However, the main disadvantage of this approach is that it takes relatively much more time compared to other approaches.

Remark 1. It is worthy to note that the cheap formulas of Rohn and Hladîk perform well when the interval matrix elements are thin (small uncertainties). These formulas may not work in the case of large uncertainties due to the warping effect (over estimation) which usually leads to a large outer bound of the interval eigenvalue. In this case, the vertex approach presents a very interesting solution to highly reduce the warping effect.

\section{INTERVAL LUENBERGER OBSERVER-BASED STATE FEEDBACK DESIGN}

Consider a linear Multi Input Multi Output (MIMO) interval uncertain system described by the following model:

$$
\left\{\begin{array}{l}
\dot{x}(t)=\boldsymbol{A} x(t)+\boldsymbol{B} u(t) \\
y(t)=\boldsymbol{C} x(t)
\end{array}\right.
$$

where $x \in R^{n}, u \in R^{m}, y \in R^{p}, \boldsymbol{A} \in I R^{n \times n}, \boldsymbol{B} \in I R^{n \times m}$, and $\boldsymbol{C} \in I R^{p \times n}$. The matrices $\boldsymbol{A}, \boldsymbol{B}, \boldsymbol{C}$ contain uncertain elements which are bounded by elements lying in known upper and lower bound; i.e. $\boldsymbol{A}=[\underline{A}, \bar{A}], \boldsymbol{B}=[\underline{B}, \bar{B}]$, and $\boldsymbol{C}=$ $[\underline{C}, \bar{C}]$.

Remark 2. Notice that even if the input command $u$ is known as non-interval signal, the model above normally yields interval state and output signals $x$ and $y$ respectively due to the interval parameters. However, in practice the real process is non-interval but assumed to have a behavior inside the above model. We therefore maintain the signals $x$ and $y$ (and $u$ ) as non-intervals.

\section{A. Interval Controller design}

Let us assume that the interval system $(\boldsymbol{A}, \boldsymbol{B}, \boldsymbol{C})$ is observable and controllable in the sense of Y. Smagina theorem for interval systems [5]. Since the direct measurement of state variables $x$ is usually impossible for many reasons (physical, economical ...), in this paper we propose to control the interval systems by a Luenberger observer-based state feedback control design.

The design of the feedback controller always necessitates a compensator (feedforward gain) to ensure the zero steadystate error. However, in the case of interval system a static feedforward gain can not be used because of the uncertainties of parameters. Therefore, an integral compensation in the loop will be employed in this paper to ensure the zero steady-state error. The proposed control law is therefore depicted in Fig.1 and given by:

$$
u(t)=-K \hat{x}+N \xi(t)
$$

where $\hat{x}$ is the estimated states by means of Luenberger observer, $K$ and $N$ are the feedback and feedforward gains respectively, and $\xi(t)$ is the integral of the tracking error (i.e. $\dot{\xi}=r(t)-y(t))$. Thus, the closed-loop system becomes:

$$
\dot{x}(t)=\boldsymbol{A} x(t)-\boldsymbol{B} K \hat{x}(t)+\boldsymbol{B} N \xi(t)
$$

It is well known that the integral action makes the closedloop system robust against slow system parameters variations. However, when the parameters are affected by fast and slow variations, the search for robust gains must be considered to ensure the stability and the desired performances of the closedloop system.

The interval version of the Luenberger observer is described by the following equations:

$$
\left\{\begin{array}{l}
\hat{\dot{x}}(t)=\boldsymbol{A} \hat{x}(t)+\boldsymbol{B} u(t)+L(y-\hat{y}) \\
=(\boldsymbol{A}-L \boldsymbol{C}-\mathbf{B} K) \hat{x}(t)+\boldsymbol{B} N \xi(t)+L \boldsymbol{C} x
\end{array}\right.
$$

Therefore, the augmented interval state-space equation can be introduced as:

$$
\begin{gathered}
\left(\begin{array}{c}
\dot{x}(t) \\
\hat{\dot{x}}(t) \\
\dot{\xi}(t)
\end{array}\right)=\left[A_{c l}\right]\left(\begin{array}{l}
x(t) \\
\hat{x}(t) \\
\xi(t)
\end{array}\right)+\left[B_{c l}\right] r(t) \\
y(t)=\left[C_{c l}\right]\left(\begin{array}{l}
x(t) \\
\hat{x}(t) \\
\xi(t)
\end{array}\right)
\end{gathered}
$$

where, $\left[A_{c l}\right]=\left(\begin{array}{ccc}\boldsymbol{A} & -\boldsymbol{B} K & \boldsymbol{B} N \\ 0 & \boldsymbol{A}-L \boldsymbol{C}-\boldsymbol{B} K & \boldsymbol{B} N \\ -\boldsymbol{C} & 0 & 0\end{array}\right)$;

$\left[B_{c l}\right]=\left(\begin{array}{l}0 \\ 0 \\ I\end{array}\right) ; \quad$ and $\quad\left[C_{c l}\right]=\left(\begin{array}{lll}C & 0 & 0\end{array}\right)$. 


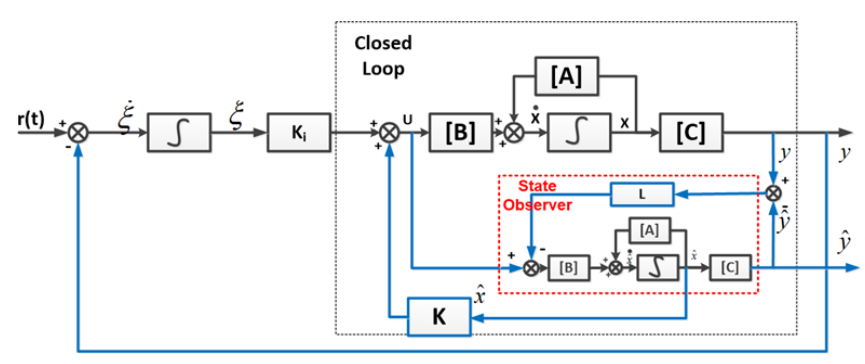

Fig. 1. The overall observer-based state feedback control schema

If we consider that $\overline{\left[A_{c l}\right]}=T\left[A_{c l}\right] T^{-1}$, by similarity transformation it is guaranteed that $\lambda\left(\overline{\left[A_{c l}\right]}\right)=\lambda\left(\left[A_{c l}\right]\right)$. Consequently, let us define $\overline{\left[A_{c l}\right]}$ as:

$$
\overline{\left[A_{c l}\right]}=T\left[A_{c l}\right] T^{-1}
$$

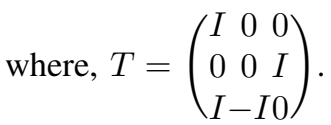

If we consider that the used system matrices to synthesize the controller and the observer are the same and belong to the interval system $(\boldsymbol{A}, \boldsymbol{B}, \boldsymbol{C})$, we get:

$$
\overline{\left[A_{c l}\right]}=\left(\begin{array}{ccc}
\boldsymbol{A}-\boldsymbol{B} K \boldsymbol{B} N & -\boldsymbol{B} K \\
-\boldsymbol{C} & 0 & 0 \\
0 & 0 & \boldsymbol{A}-L \boldsymbol{C}
\end{array}\right)
$$

By employing matrix manipulations in (8), we find:

$$
\begin{gathered}
\operatorname{det}\left(\overline{\left[A_{c l}\right]}\right)=\left(\begin{array}{ccc}
S I-\boldsymbol{A}+\boldsymbol{B} K-\boldsymbol{B N} & \boldsymbol{B} K \\
\boldsymbol{C} & S I & 0 \\
0 & 0 & S I-\boldsymbol{A}+L \boldsymbol{C}
\end{array}\right) \\
=\operatorname{det}\left(\begin{array}{cc}
S I-\boldsymbol{A}+\boldsymbol{B} K-\boldsymbol{B} N \\
\boldsymbol{C} & S I
\end{array}\right) \operatorname{det}(S I-\boldsymbol{A}+L \boldsymbol{C})
\end{gathered}
$$

This is known in the literature as the separation theorem which is widely applied to non-interval systems to find separately the gains for the controller and the observer [17].

\section{B. Problem formulation using Set-Inversion}

The robust observer-based state feedback control design can be outlined by finding the set of gain matrix $[K],[L]$ and $[N]$ (defined previously) that assign the system eigenvalues to a desired region taking into account the uncertainty of the interval system. Therefore, the problem arises in finding the robust gains of the closed-loop system when the inclusion of (10) is satisfied.

$$
\text { eig }\left[\overline{\left[A_{c l}\right]}\right] \subseteq \Omega_{\text {Desired region }}
$$

where $\overline{\left[A_{c l}\right]}$ is the augmented closed-loop matrix of the system (8) and $\Omega_{\text {Desiredregion }}$ is the desired subregions for the interval eigenvalues.

With the help of the separation theorem (9) the inclusion problem (10) can be divided into two inclusion problems, one for the feedback controller and the other for the observer as demonstrated by the following equations:

$$
\text { eig }\left[\left(\begin{array}{cc}
S I-\boldsymbol{A}+\boldsymbol{B} K-\boldsymbol{B N} \\
\boldsymbol{C} & S I
\end{array}\right)\right] \subseteq \Omega_{\text {Desiredregion-controller }}
$$

$$
\text { eig }[(S I-\boldsymbol{A}+L \boldsymbol{C})] \subseteq \Omega_{\text {Desired region-oberever }}
$$

The interval eigenvalues of the observer system have to be placed so that the state estimator $\widehat{x}$ can be made to approach $x$ faster than the feedback control system by at least four times. Thus, we propose to set the desired regions of the observer $\Omega_{\text {Observer }}$ and the controller $\Omega_{\text {controller }}$ as depicted in Fig.2.

Remark 3. In fact, it is not practical to find a set robust gains that can assign all eigenvalues of the interval control system in the desired region $\Omega_{\text {controller }}$ shown in Fig.2. However, the set of gains should guarantee that the dominant eigenvalues of the control system are within the region $\Omega_{\text {controller }}$.

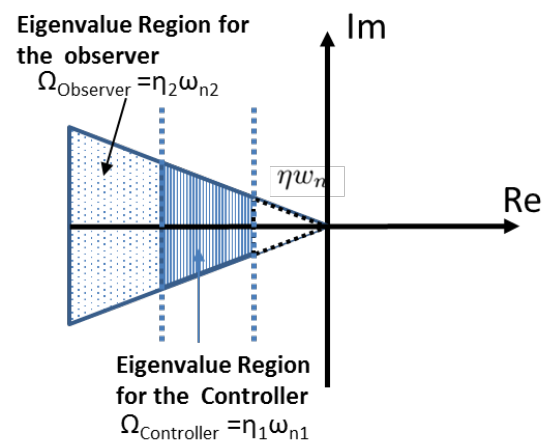

Fig. 2. Desired regions for the controller and the observer in the complex plane.

This problem of interval eigenvalues assignment can be transformed into a set-inversion problem that can be solved using inversion algorithms. The set inversion operation consists in searching the reciprocal image $X$ of a compact set $Y$ by a function $f$, i.e. $X=f^{-1}(Y)=\left\{X \in R^{n} \mid f(x) \in Y\right\}$ [3], [18]. The SIVIA algorithm, introduced by Jaulin in 1993 [18], is one of the powerful approach existing in the literature. This algorithm is based on the subpaving technique which uses a set of non-overlapping boxes to approximate the solution set of the inversion problems. In this paper, the SIVIA algorithm is adapted to approximate with subpaving the set solutions $[K],[L]$ and $[N]$ that satisfy the inclusions (11) and (12). The proposed algorithm is outlined in Table.1.

The recursive SIVIA-based algorithm requires as input the interval system matrices $([A],[B],[C])$, initial box for the gains $\left[\Theta_{0}\right]$ that may contain the solutions, the desired region of the eigenvalues, and the desired precision for the subpaving $\epsilon$. The interval eigenvalues computation can be performed using the Hladik formula or the vertex approach depending on the thickness of the interval parameters as explained in section.II. The proposed algorithm provides a complete information about the ranges of feedback gains including: $\left[\Theta_{i n}\right]$ inner (solution), $\left[\Theta_{\text {out }}\right]$ outer (undefined), and $\left[\Theta_{\text {Inf }}\right]$ infeasible (no solution) subpavings. 
TABLE I

THE PROPOSED RECURSIVE SIVIA-BASED ALGORITHM.

\begin{tabular}{|l|l|}
\hline & $\begin{array}{l}\text { SIVIA (in: }[A],[B],[C],\left[\Theta_{0}\right], \epsilon, \Omega_{\text {desired region }} ; \\
\left.\text { out: }\left[\Theta_{\text {in }}\right],\left[\Theta_{\text {out }}\right],\left[\Theta_{\text {Infeasibl }}\right]\right)\end{array}$ \\
\hline 1 & - Compute $A_{\text {closedloop }}([A],[B],[C],[\Theta])$ \\
2 & - Estimate the interval eigenvalue eig $\left(\left[A_{\text {closedloop }}\right]\right)$ \\
3 & - If eig $\left(\left[A_{\text {closedloop }}\right]\right) \subseteq \Omega_{d r}$ Then $\left[\Theta_{\text {in }}\right]=\left[\Theta_{\text {in }}\right] \cup[\Theta]$ return; \\
4 & - Elself eig $\left(\left[A_{\text {closedloop }}\right]\right) \cap \Omega_{d r}=\oslash$ Then \\
5 & {$\left[\Theta_{\text {Inf }}\right]=\left[\Theta_{\text {Inf }}\right] \cup[\Theta]$} \\
6 & - Elself $[\Theta]<\epsilon$ Then $\left[\Theta_{\text {out }}\right]=\left[\Theta_{\text {out }}\right] \cup[\Theta]$ return; \\
7 & - Else bisect $[\Theta]$ and stack the two resulting boxes $\left[\Theta_{n}\right]$ and $\left[\Theta_{n+1}\right]$. \\
& - If stack $\neq \oslash$ then unstack into $\left[\Theta_{n+1}\right] ;$ Else End. \\
\hline
\end{tabular}

Remark 4. The recursive algorithm, outlined in Table.1, is used to find the robust gains matrices for both the observer system and the controller system. In both cases we adapt the algorithm such that: in the case of the observer synthesis, the set $[\Theta]$ represents the observer gains matrix $[L], \Omega_{d r}$ is the desired region of the observer $\Omega_{\text {Observer }}$, as depicted in Fig.2, where $A_{\text {closedloop }}$ is given by (12). Whereas, for the case of the feedback control synthesis the set $[\Theta]$ represents the gains matrix $[[K][N]]$, where $A_{\text {closedloop }}$ is given by (11) and $\Omega_{d r}$ is the desired region of the controller $\Omega_{\text {controller }}$.

In the remaining part of this paper, we will use the proposed robust interval observer-based state feedback synthesis to design a robust controller for a multi-DOF micropositioning system. However, the proposed control synthesis can be applied to any system that can be modeled by interval statespace model and that satisfies the observability/controllability conditions.

\section{EXPERIMENTAL SETUP AND SYSTEM IDENTIFICATION}

\section{A. Experimental apparatus}

In this section we introduce an improved version of the 5DOF positioning system which has been presented in [19]. The proposed positioner is based on a monolithic skeleton passivestructure equipped with six piezostacks actuators. Thanks to 3D printing technologies, such construction reduces assembly operation and consequently allows to vanish mechanical plays that may affect displacement resolution. The structure is able to perform 5-DOF: three translations along X-Y-Z axes and two rotations about $X-Y$ axes where single-beam and crossbeam are used to guide linear and angular displacement, as depicted by the CAD model given in Fig.3-b.

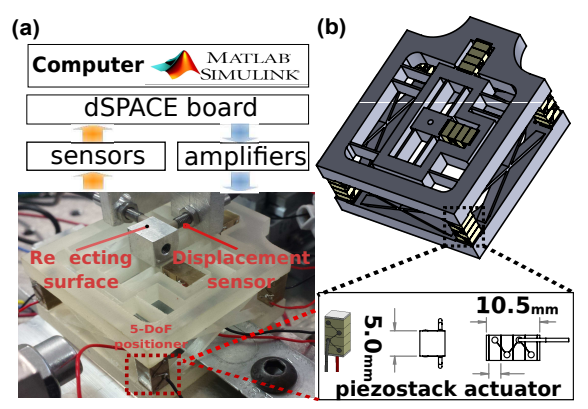

Fig. 3. a-Presentation of the experimental set-up. b-3D CAD model of the proposed positioner based on a monolithic passive structures driven by six piezostack actuators.
The experimental setup depicted in Fig.3-a is composed of:

- the 5-DOF prototype positioner,

- three displacement inductive sensors (ECL202 from IBS company) with a resolution of $40 \mathrm{~nm}$ and a bandwidth of $15 \mathrm{kHz}$.

- an acquisition board which includes a computer and a converter-board (DS1103 from dSPACE company).

- three voltage amplifiers (A400DI from FLC Electronics company). Each amplifier has two independent channels able to provide a supply voltage up to $\pm 200 \mathrm{~V}$.

Due to the lack of high precision sensors to capture the orientations of the positioner, in the sequel we consider only the $\mathrm{X}, \mathrm{Y}$ and $\mathrm{Z}$ displacements (i.e. three degrees of freedom: 3-DOF) of the positioning system to validate the proposed control strategy.

\section{B. System identification}

The nonlinear characteristics of the used piezoelectric actuators ( i.e. hysteresis, creep,...) and the sensitivity of the micropositioning system to the environment variations at this scale may affect its behavior during the micro/nano applications. Unfortunately, it is extremely difficult to characterize and to model the change of the environment and its effects on the system during the experimentation. Therefore, in this paper we propose to employ the interval state-space models to model the 3-DOF micropositioning stage by considering these effects as parametric uncertainties and by just bounding them with intervals [20], [3], [4]. Thus, to obtain the interval statespace model, we start by identifying the non-interval model of the system then we will explain how to obtain the interval model in the end of this subsection.

To characterize the 3-DOF actuator, we apply first a step voltage $U_{x}$ of an amplitude $75 \mathrm{~V}$ and we set $U_{y}$ and $U_{z}$ to zero and capture the displacement along $X, Y$, and $Z$ axes which are signed by $\sigma_{x}, \sigma_{y}$, and $\sigma_{z}$ respectively. Then we repeat the same procedures with $U_{y}$ and $U_{z}$. Basically, the transfer function $G(s)$ to be identified for the 3-DOF micropositioning stage is composed of nine transfer functions as given in (13).

$$
G(s)=\left(\begin{array}{l}
G_{x x}(s) G_{y x}(s) G_{z x}(s) \\
G_{x y}(s) G_{y y}(s) G_{z y}(s) \\
G_{x z}(s) G_{y z}(s) G_{z z}(s)
\end{array}\right)
$$

In order to identify the different transfer functions that describe the dynamics of the system along the different axes (13), we use the Box-Jenkins method, which is already available in the System Identification MatlabToolbox. Particularly, a second order model for all transfer functions have been chosen in our case because they are sufficient to represent the dynamics of the positioning stage. Therefore, the identified transfer functions are found as: 


$$
\begin{gathered}
G_{x x}(s)=\frac{25.84 s+3.93 e 05}{s^{2}+2669 s+3.471 e 06} \\
G_{y y}(s)=\frac{21.43 s+1.806 e 05}{s^{2}+1666 s+1.568 e 06} \\
G_{z z}(s)=\frac{-9.862 s+1.548 e 04}{s^{2}+578.9 s+1.29 e 05} \\
G_{z x}(s)=\frac{1.293 s+969.7}{s^{2}+399.6 s+4.153 e 05} \\
G_{z y}(s)=\frac{12.26 s-5547}{s^{2}+5.587 e-08 s+8.696 e 05} \\
G_{x y}(s)=\frac{-9.116 s-124.859}{s^{2}+1.062 e 03 s+1.499 e 05} \\
G_{y x}(s)=\frac{13.800 s-0.3789}{s^{2}+1274.79 s+2891.46} \\
G_{x z}(s)=\frac{1.357 s-100.38}{s^{2}+401.347 s+4.291 e 05} \\
G_{y z}(s)=\frac{1.2058 s-561.11}{s^{2}+5.789 e-08 s+8.959 e 05}
\end{gathered}
$$

The structure of the proposed micropositioner reduces the cross-coupling effects because the different axes are structurally independent. This can be seen clearly from the static gains of the cross-coupling transfer functions in (14) (i.e. $s=0$ ). Therefore, we can neglect the cross-coupling effects between the different axes especially at low frequencies. However, during the experimentation test, we found that when we apply an input voltage on $\mathrm{Z}$ axis (i.e. on the four parallel piezostacks) the micropositioning system has a considerable cross-coupling on the $X$ and $Y$ axes which has to be considered. This cross-coupling effect is caused by the unlike reactions of the four parallel piezostacks actuators when the input voltage is applied and it can be seen clearly from the static gains of the $G_{z y}(s)$ and $G_{z x}(s)$ in (14). Therefore, in the final model only the cross-coupling transfer functions $G_{x y}(s), G_{x z}(s), G_{y x}(s)$, $G_{y z}(s)$ are set to zero, as presented in (15).

$$
G(s)=\left(\begin{array}{ccc}
G_{x x}(s) & 0 & G_{z x}(s) \\
0 & G_{y y}(s) & G_{z y}(s) \\
0 & 0 & G_{z z}(s)
\end{array}\right)
$$

To obtain the interval model, we propose to consider each parameter of (15) as center and we add a radius of $10 \%$. Thus:

$$
\begin{aligned}
G_{x x}(s) & =\frac{\left[b_{11}\right] s+\left[b_{12}\right]}{s^{2}+\left[a_{11}\right] s+\left[a_{12}\right]} \\
G_{y y}(s) & =\frac{\left[b_{21}\right] s+\left[b_{22}\right]}{s^{2}+\left[a_{21}\right] s+\left[a_{22}\right]} \\
G_{z z}(s) & =\frac{\left[b_{31}\right] s+\left[b_{32}\right]}{s^{2}+\left[a_{31}\right] s+\left[a_{32}\right]} \\
G_{z x}(s) & =\frac{\left[b_{41}\right] s+\left[b_{42}\right]}{s^{2}+\left[a_{41}\right] s+\left[a_{42}\right]} \\
G_{z y}(s) & =\frac{\left[b_{51}\right] s+\left[b_{52}\right]}{s^{2}+\left[a_{51}\right] s+\left[a_{52}\right]}
\end{aligned}
$$

where

$$
\begin{array}{lll}
{\left[b_{11}\right]=[23.2560,28.4241]} & ;\left[a_{11}\right]=[2.4020,2.9360] \times 10^{3} ; \\
{\left[b_{12}\right]=[3.5369,4.3231] \times 10^{5}} & ;\left[a_{12}\right]=[3.1238,3.8182] \times 10^{6} ; \\
{\left[b_{21}\right]=[19.2870,23.5731]} & ;\left[a_{21}\right]=[1.4993,1.8327] \times 10^{3} ; \\
\left.\left[b_{22}\right]=[1.6253,1.9867] \times 10^{5}\right) & ;\left[a_{22}\right]=[1.4111,1.7249] \times 10^{6} ; \\
{\left[b_{31}\right]=[-10.8483,-8.8757]} & ;\left[a_{31}\right]=[521.0099,636.7901] \\
\left.\left[b_{32}\right]=[1.3931,1.7029] \times 10^{4}\right) & ;\left[a_{32}\right]=[1.1609,1.4191] \times 10^{5} ; \\
{\left[b_{41}\right]=[1.1636,1.4224]} & ;\left[a_{41}\right]=[359.6399,439.5601] \\
\left.\left[b_{42}\right]=[0.8727,1.0667] \times 10^{3}\right) & ;\left[a_{42}\right]=[3.7376,4.5684] \times 10^{5} ; \\
{\left[b_{51}\right]=[11.0340,13.4861]} & ;\left[a_{51}\right]=[0.5028,0.6146] \times 10^{-7} ; \\
{\left[b_{52}\right]=[-6.1018,-4.9922] \times 10^{3} ;\left[a_{52}\right]=[7.8263,9.5657] \times 10^{5} ;}
\end{array}
$$

In fact, in [3], it is shown that the $10 \%$ of radius is sufficient enough to describe the uncertainties of numerous cases of piezoelectric actuators. This margin presents a good compromise between the widths of parameter uncertainties and the chance to find the robust gains for the closed-loop. Furthermore, according to the performances inclusion system [21], the obtained robust gains will guarantee the performances for any uncertainties within the interval, which was created to have $10 \%$ radius from the center.

The above-mentioned interval MIMO transfer function model of the micropositioning system can be expressed by the following interval state-space model using canonical transformation [22], [23]:

$$
\left\{\begin{array}{l}
\dot{x}(t)=\boldsymbol{A} x(t)+\boldsymbol{B} u(t) \\
y(t)=\boldsymbol{C} x(t)
\end{array}\right.
$$

where

$$
\begin{gathered}
\boldsymbol{A}=\left[\begin{array}{ccc}
\boldsymbol{A}_{x x} & 0 & \boldsymbol{A}_{z x} \\
0 & \boldsymbol{A}_{y y} & \boldsymbol{A}_{z x} \\
0 & 0 & \boldsymbol{A}_{z z}
\end{array}\right] \\
\boldsymbol{B}=\left[\begin{array}{ccc}
\boldsymbol{B}_{x x} & 0 & 0 \\
0 & \boldsymbol{B}_{y y} & 0 \\
\boldsymbol{B}_{z x} & \boldsymbol{B}_{z y} & \boldsymbol{B}_{z z}
\end{array}\right]^{t} ; \boldsymbol{C}=\left[\begin{array}{ccc}
\boldsymbol{C}_{x x} & 0 & \boldsymbol{C}_{z x} \\
0 & \boldsymbol{C}_{y y} \boldsymbol{C}_{z y} \\
0 & 0 & \boldsymbol{C}_{z z}
\end{array}\right] \\
\text { V. CALCUlation OF THE Robust } \\
\text { OBSERVER/CONTROLLER GAINS }
\end{gathered}
$$

The goal of the robust observer-based state feedback control synthesis is to find the robust gains that ensure the stability and the desired performances of the observer and the feedback controller under system uncertainties.

Indeed, by using the separation principle introduced previously (9), we get:

$\operatorname{det}\left(\overline{\left[A_{c l}\right]}\right)=\operatorname{det}\left(\begin{array}{cc}S I-\boldsymbol{A}+\boldsymbol{B} K-\underset{\boldsymbol{B} N}{\boldsymbol{C}} & S I\end{array}\right) \operatorname{det}(S I-\boldsymbol{A}+L \boldsymbol{C})$

Based on the structure of the state-space model of the interval system (17), we consider the structures of the gains matrices $K, N$, and $L$ as follows:

$$
\begin{aligned}
K & =\left(\begin{array}{ccc}
K_{x x} & 0 & K_{z x} \\
0 & K_{y y} & K_{z y} \\
0 & 0 & K_{z z}
\end{array}\right) ; N=\left(\begin{array}{ccc}
N_{x} & 0 & 0 \\
0 & N_{y} & 0 \\
0 & 0 & N_{z}
\end{array}\right) ; \\
L & =\left(\begin{array}{ccc}
L_{x x} & 0 & L_{z x} \\
0 & L_{y y} & L_{z y} \\
0 & 0 & L_{z z}
\end{array}\right)
\end{aligned}
$$

In order to obtain the robust gains for the feedback controller and the observer, the interval state-space model of the multi-DOF positioning system (17) is used with the help of 
the proposed recursive SIVIA-based algorithm (Table.1). Foremost, we set the desired performances for both the feedback controller and the observer in the time-domain such that the state estimation is made at least four times faster than the feedback controller. Furthermore, in micro/nano manipulation and assembly applications, the rapidity is highly required and the overshoot is extremely undesirable because it may cause micro/nano objects damage. Therefore we set the desired performances of the step response behavior as follows: a negligible overshoot and settling times $T_{s 1} \leq 8 \mathrm{~ms}$ for the observer system and $T_{s 2} \leq 40 \mathrm{~ms}$ for the controller system. Consequently, let us define the desired regions $\Omega_{\text {controller }}$ and $\Omega_{\text {observer }}$ as follows: for the the controller we set $\xi_{1}=$ $\eta_{1} \cdot \omega_{n 1}=115$ and $\theta=55,7^{\circ}$, and for the observer we set $\xi_{2}=\eta_{2} \cdot \omega_{n_{2}}=575.6$, where $\eta$ and $\omega_{n}$ are the damping ratio and the natural frequency respectively [24].

1) Search for feedback controller gains: As explained previously, the search for the robust gains for the observer and the control systems can be performed separately by means of the separation principle. Indeed the controller gains are related directly to the following determinant matrix:

$$
\operatorname{det}\left(\begin{array}{cc}
S I-\boldsymbol{A}+\boldsymbol{B} K-\boldsymbol{B N} \\
\boldsymbol{C} & S I
\end{array}\right)
$$

By replacing the interval system matrices in (18) and by using matrix algebraic properties, the search process for the robust gains for the controller is simplified to the search of the robust gains for three subsystems in a separated way as described by the following equation:

$$
\begin{array}{r}
\operatorname{det}\left(\begin{array}{c}
S I-\boldsymbol{A}+\boldsymbol{B} K-\boldsymbol{B} N \\
\boldsymbol{C}
\end{array}\right)= \\
\operatorname{det}\left(\begin{array}{cc}
S I-\boldsymbol{A}_{x x}-\boldsymbol{B}_{x x} K_{x x} \boldsymbol{B}_{x x} N_{x} \\
-\boldsymbol{C}_{x x} & S I
\end{array}\right) \\
\operatorname{det}\left(\begin{array}{cc}
S I-\boldsymbol{A}_{y y}-\boldsymbol{B}_{y y} K_{y y} \boldsymbol{B}_{y y} N_{y} \\
-\boldsymbol{C}_{y y} & S I
\end{array}\right) \\
\operatorname{det}\left(\begin{array}{cc}
S I-\boldsymbol{A}_{z z}-\boldsymbol{B}_{z z} K_{z z} \boldsymbol{B}_{z z} N_{z} \\
-\boldsymbol{C}_{z z} & S I
\end{array}\right)
\end{array}
$$

To find the set solution $[K]$ and $[N]$ of each subsystem, we use the proposed recursive SIVIA-based algorithm described in Table.1. Foremost, we choose an initial box $\left[K_{i 1}\right] \times\left[K_{i 2}\right] \times\left[N_{i}\right]=\left[-5 \times 10^{1}, 5 \times 10^{1}\right] \times[-5,5] \times\left[-1 \times 10^{3}, 1 \times 10^{3}\right]$, where $i$ belongs to $\{x x, y y, z z\}$, which corresponds to the three subsystems given in (19). Also we set the accuracy of subpaving to $\epsilon=0.01$ and we define $\Omega_{\text {controller }}$ as the desired region for the interval eigenvalues. The obtained subpaving results of each subsystem are depicted in Fig.4 (a, b and c). The red boxes correspond to the inner subpavings $\left[K_{i n}\right]$, i.e. the set solutions $\left[\left[K_{i 1}\right]\left[K_{i 2}\right]\left[N_{i}\right]\right]$ that satisfy the inclusion (11). The white boxes correspond to the subpavings $\left[K_{\text {Unfeasible }}\right]$ where the inclusion condition (11) is not satisfied. The yellow boxes refer to $\left[K_{\text {out }}\right]$ where no decision on the inclusion is taken.

2) Search for observer gains: In order to find the robust gains for the observer system, we follow the same procedures detailed previously for the controller. In this time we use the following determinant matrix (20) that corresponds to the observer system, obtained from the separation principle.

$$
\operatorname{det}(S I-\boldsymbol{A}+L \boldsymbol{C})
$$

Moreover, the determinant matrix (20) can be divided by itself to three subsystems using matrix simplification as we did for the controller. The obtained subpaving results of each subsystem are depicted in Fig.4 (d, e and f).

\section{A. Simulation results with Monte-Carlo technique}

Before validating experimentally the proposed controller design, we start by testing the robustness of the obtained gains of the observer and the feedback controller in simulation by means of Monte-Carlo technique. During the simulation validation, we select randomly the robust gains for the observer and the controller from the solution boxes depicted in Fig.4. Consequently, we set the gains for the observer and the controller as $L_{x x}=[50,5], L_{y y}=[50,5], L_{z z}=[0.2,100]$, $\left[K_{x x}, N_{x x}\right]=[1,0.05,1200],\left[K_{y y}, N_{y y}\right]=[1,0.05,1200]$, $\left[K_{z z}, N_{z z}\right]=[0.2,-10,800]$, where the gains $L_{z x}, L_{z y}$, $K_{z x}$, and $K_{z y}$ are set to zeros. Afterwards we start simulation using Monte-Carlo in which we select randomly a value for the system matrices inside the interval system $([A],[B],[C])$ and each time we draw the obtained eigenvalues, as depicted in Fig.5. The results clearly show that the observer and the controller system are always stable and satisfy the desired performances related to the damping ratio and the natural frequency $\left(\xi_{1}, \xi_{2}\right.$, and $\theta$ defined previously) as well as the system matrices are inside the interval system $([A],[B],[C])$, which means that the observer and the controller are robust against parameters uncertainties.

The step responses for the closed-loop system using MonteCarlo simulation are depicted in Fig.6. We notice that all the step responses of the closed-loop system satisfy the desired performances with a negligible overshoot (1\%) and with a settling time $T_{s} \leq 40 \mathrm{~ms}$. Moreover, it can be seen that the closed-loop system rapidly rejects the cross-coupling effect with a time less than $40 \mathrm{~ms}$.

\section{EXPERIMENTAL VALIDATION}

The previous sections were devoted to design the robust observer-based state feedback controller in which the robust gains for the observer and the controller are calculated and tested in simulation. In this subsection, the designed observer and controller are implemented and tested experimentally.

Figure 7 represents the experimental bode diagrams of the identified transfer functions of the closed-loop system $\left(T_{x x}\right.$, $T_{y y}, T_{z z}, T_{z x}$, and $T_{z y}$ ) also the open-loop transfer functions $\left(G_{x x}, G_{y y}, G_{z z}, G_{z x}\right.$, and $\left.G_{z y}\right)$. This figure demonstrates that the closed-loop system achieves a convenient bandwidth relative to the bandwidth of the open-loop system for $H_{x x}$, $H_{y y}$, and $H_{z z}$. Moreover, from the bode diagram of the crosscouplings transfer functions $H_{z x}$ and $H_{z y}$, we can see that the closed-loop system ensures the rejection of the cross-coupling effect in low and relatively high frequencies, whereas, for the low frequencies the rejection has a very small amplitude compared with high frequencies. Furthermore, it is worthy to note that the cross-coupling $H_{z x}$ and $H_{z y}$ are larger than 


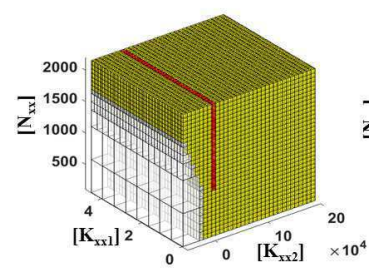

(a)

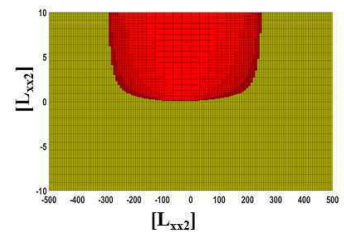

(d)

Inner (Solution)

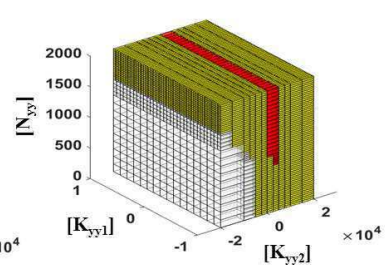

(b)

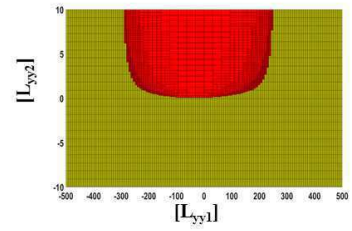

(e)

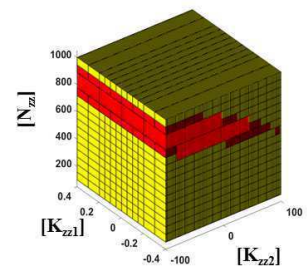

(c)

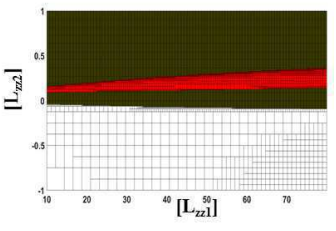

(f)

Fig. 4. Resulting solution gains for the controller and the observer.

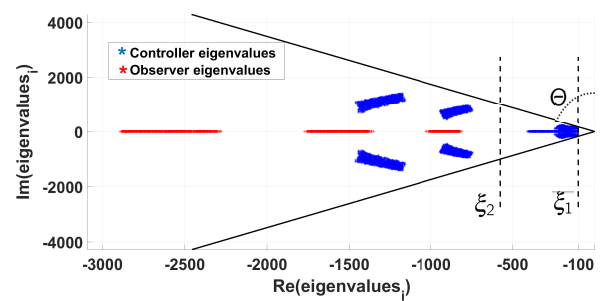

Fig. 5. Draw of the eigenvalues of the observer and the controller (closed-loop system) in the complex plan using Monte-Carlo techniques.

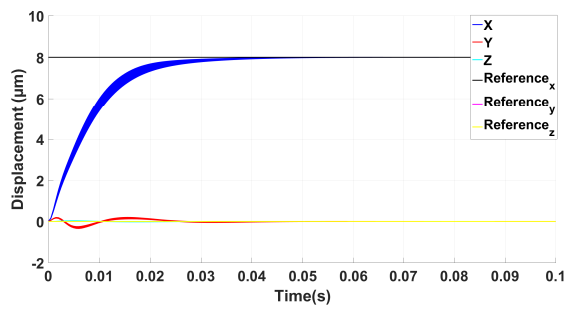

Fig. 6. Step responses of the closed-loop system using Monte-Carlo technique.

that of open-loop after about $500 \mathrm{rad} / \mathrm{s}$ which is acceptable bandwidth for low speed applications. In the other side, the cross-coupling $H_{y x}, H_{x y}, H_{x z}, H_{y z}$ are remain negligible in closed-loop for high and low frequencies as shown in fig.7.

In order to test the tracking performances of the closedloop system, we carry out an experimental test with various trajectories including helix and sine wave trajectories with different frequencies. However to evaluate the accuracy of the tracking, in the sequel we will use the rms (root-mean-square) error criteria which is calculated from the following equation:

$$
r m s=\sqrt{\left(x_{r e f}-x\right)^{2}+\left(y_{r e f}-y\right)^{2}+\left(z_{r e f}-z\right)^{2}}
$$

The tracking result of a sinusoidal trajectory applied on the three axes with same frequencies is depicted in Fig.8-a which represents a line in the cartesian space. In fact, two experiment tests were performed. The first one used a low frequency of 1
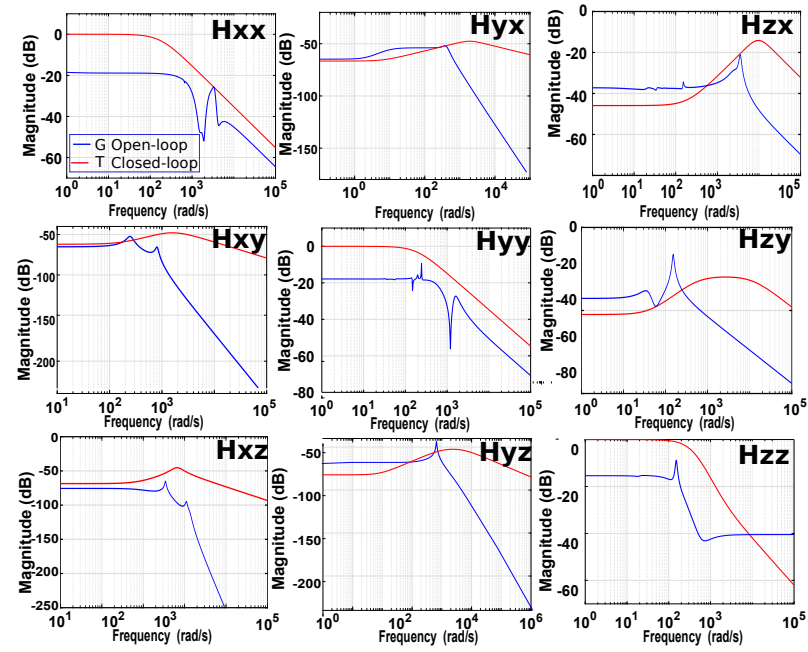

Fig. 7. The open-loop and closed-loop frequency responses of the positioning system (experimentation).

$\mathrm{rad} / \mathrm{sec}$ in which the positioning system tracks successfully the desired trajectory with rms error (21) of $0.06 \mu \mathrm{m}$ that represents approximately $0.6 \%$ of the full range as shown in Fig.8-c. The second one the frequencies of the input signals are increased to $12 \mathrm{rad} / \mathrm{sec}$, the $\mathrm{rms}$ error relatively increases to $0.27 \mu \mathrm{m}$ ( $3 \%$ of the full range) as depicted in Fig.8-d. This error refers to the phase-lag phenomena and hysteresis behavior that characterize the dynamics of the piezoelectric systems at high frequencies. However, the errors are convenient for multi-DOF micro/nano positioning applications especially in low-speed conditions. For a high-speed tracking conditions, we propose as future work to combine the proposed approach in this paper with resonant control techniques to obtain a high tracking performances [25]. Furthermore, we need to make some improvements on the micro-positioning stage itself and also a more sophisticated sensors need to be used.

Figure 8-b presents a helix trajectory test where the positioning system is forced to track a helix shaped trajectory by applying simultaneously a sine waves of amplitude $5 \mu \mathrm{m}$ on 


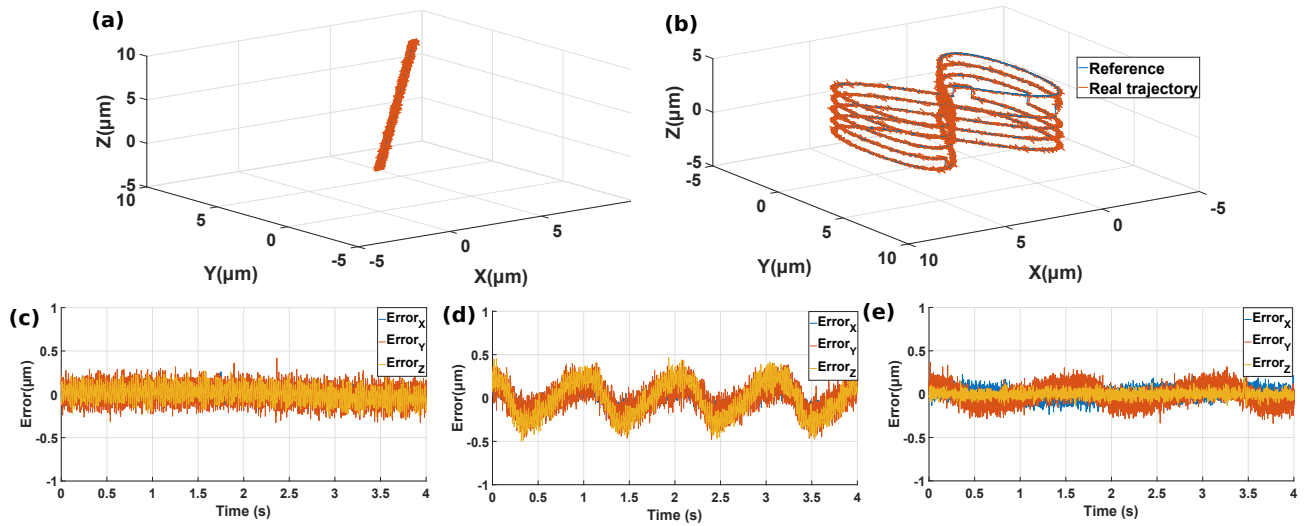

Fig. 8. Sinusoidal and Helix trajectory tests: a- Sinusoidal trajectory in the cartesian space,b- Helix trajectory, c- the obtained error for the sinusoidal trajectory at frequency of $1 \mathrm{rad} / \mathrm{sec}$ for $\mathrm{X}, \mathrm{Y}$, and $\mathrm{Z}$ axes, d- the obtained error for frequencies of $12 \mathrm{rad} / \mathrm{sec}$. e- the obtained error for the Helix trajectory.

$\mathrm{X}$ and $\mathrm{Y}$ axes with frequencies of $2 \mathrm{rad} / \mathrm{sec}$ and $4 \mathrm{rad} / \mathrm{sec}$, respectively, and series of steps on $\mathrm{Z}$ axes. Indeed, it is shown that the helix trajectory tracking is successfully achieved, with a tracking rms error less than $0.2 \mu \mathrm{m}$ for $\mathrm{X}, \mathrm{Y}$ and $\mathrm{Z}$ axis (less than $2 \%$ of the full range).

\section{CONCLUSIONS}

In this paper, a robust observer-based state feedback control design is introduced to control a multi-DOF micropositioning system. The proposed robust control synthesis is based on interval analysis combined with regional pole assignment technique using interval eigenvalue computation. Indeed, we propose a recursive algorithm, called recursive SIVIA-based algorithm, to find the robust gains for the controller and the observer under system uncertainties. Simulation validation using Monte-Carlo technique is performed to validate the proposed approach under system uncertainties. Furthermore, an experimental validation was carried out to control a new multi-DOF positioning system. These experimental results validated the proposed observer-based state feedback design and demonstrated the effectiveness of the proposed control synthesis to handle the problem of system uncertainties.

\section{ACKNOWLEDGMENT}

This work was supported by the French 'Investissements d'Avenir' program, project ISITE-BFC (ANR-15-IDEX-03).

\section{REFERENCES}

[1] M. Rakotondrabe, Smart Materials Based Actuators at the Micro/NanoScale. Characterization, Control and Applications. SPRINGER VERLAG, Jan. 2013.

[2] S. Khadraoui, M. Rakotondrabe, and P. Lutz, "Design of a fixed-order rst controller for interval systems: Application to the control of piezoelectric actuators," Asian Journal of Control, vol. 15, no. 1, pp. 142-154, 2013.

[3] - "Interval modeling and robust control of piezoelectric microactuators," Control Systems Technology, IEEE Transactions on, vol. 20, no. 2, pp. 486-494, 2012.

[4] _ - "Combining h-inf approach and interval tools to design a low order and robust controller for systems with parametric uncertainties: application to piezoelectric actuators," International Journal of control, vol. 85, no. 3, pp. 251-259, 2012.

[5] Y. Smagina and I. Brewer, "Using interval arithmetic for robust state feedback design," Systems \& Control Letters, vol. 46, no. 3, pp. 187 194, 2002.
[6] B. M. Patre and P. J. Deore, "Robust state feedback for interval systems: An interval analysis approach." Reliable Computing, vol. 14, no. 1, pp. 46-60, 2010.

[7] M. L. Prado, A. D. Lordelo, and P. A. Ferreira, "Robust pole assignment by state feedback control using interval analysis," in World Congress, vol. 16 , no. 1,2005 , pp. 951-951.

[8] L. Jaulin, Applied interval analysis: with examples in parameter and state estimation, robust control and robotics. Springer Science \& Business Media, 2001, vol. 1.

[9] A. Deif, "The interval eigenvalue problem," ZAMM-Journal of Applied Mathematics and Mechanics/Zeitschrift für Angewandte Mathematik und Mechanik, vol. 71, no. 1, pp. 61-64, 1991.

[10] L. Kolev and S. Petrakieva, "Assessing the stability of linear timeinvariant continuous interval dynamic systems," Automatic Control, IEEE Transactions on, vol. 50, no. 3, pp. 393-397, 2005.

[11] M. Hladík, "Bounds on eigenvalues of real and complex interval matrices," Applied Mathematics and Computation, vol. 219, no. 10, pp. 5584-5591, 2013

[12] G. Mayer, "A unified approach to enclosure methods for eigenpairs," ZAMM-Journal of Applied Mathematics and Mechanics/Zeitschrift für Angewandte Mathematik und Mechanik, vol. 74, pp. 115-128, 1994.

[13] H.-S. Ahn, K. L. Moore, and Y. Chen, "Monotonic convergent iterative learning controller design based on interval model conversion," Automatic Control, IEEE Transactions on, vol. 51, pp. 366-371, 2006.

[14] J. Rohn, "A handbook of results on interval linear problems," Internet text available at http://www. cs. cas. cz/rohn/handbook, 2005.

[15] M. T. Hussein, "Assessing 3-d uncertain system stability by using matlab convex hull functions," International Journal of Advanced Computer Science and Applications,(IJACSA), vol. 2, no. 6, 2011.

[16] S. Bhattacharyya, H. Chapellat, and L. Keel, "Robust control: the parametric approach," Upper Saddle River, 1995.

[17] K. Zhou, J. C. Doyle, and K. Glover, Robust and Optimal Control. Upper Saddle River, NJ, USA: Prentice-Hall, Inc., 1996.

[18] L. Jaulin and E. Walter, "Set inversion via interval analysis for nonlinear bounded-error estimation," Automatica, vol. 29, pp. 1053-1064, 1993.

[19] A. Mohand-Ousaid, D. Gendreau, P. Rougeat, and M. Rakotondrabe, "Design, static modeling and simulation of a 5-dof precise piezoelectric positioner," in SPIE Commercial+ Scientific Sensing and Imaging. International Society for Optics and Photonics, 2016.

[20] S. Khadraoui, M. Rakotondrabe, and P. Lutz, "Interval force/position modeling and control of a microgripper composed of two collaborative piezoelectric actuators and its automation," International Journal of Control, Automation and Systems, vol. 12, no. 2, pp. 358-371, 2014.

[21] M. Rakotondrabe, "Performances inclusion for stable interval systems," in American Control Conference (ACC), 2011. IEEE, 2011.

[22] T. Kailath, Linear systems, ser. Prentice Hall information and system sciences series. Englewood Cliffs: Prentice-Hall, 1980.

[23] K. Premaratne, E. Jury, and M. Mansour, "Multivariable canonical forms for model reduction of 2-d discrete time systems," IEEE transactions on circuits and systems, vol. 37, no. 4, pp. 488-501, 1990.

[24] R. C. Dorf and R. H. Bishop, "Modern control systems," 1998.

[25] B. Bhikkaji and S. R. Moheimani, "Integral resonant control of a piezoelectric tube actuator for fast nanoscale positioning," IEEE/ASME Transactions on mechatronics, vol. 13, no. 5, pp. 530-537, 2008. 